TEMAS DE ACTUALIDAD Rev Chil Salud Pública 2013; Vol 17 (3): 218-223

\section{MÉTODOS MIXTOS: UNA APROXIMACIÓN A SUS VENTAJAS Y LIMITACIONES EN LA INVESTIGACIÓN DE SISTEMAS Y SERVICIOS DE SALUD}

Mixed Methods: a ReView Of their advantages AND

LIMITATIONS IN HEALTH SYSTEMS AND SERVICES RESEARCH

\title{
INTRODUCCIÓN
}

La investigación en servicios de salud busca obtener información válida y confiable para tomar decisiones de cómo organizar y financiar de manera eficiente y aceptable los sistemas de salud, teniendo como preocupación central el servicio sanitario y la población beneficiada. El análisis involucra un nivel macro correspondiente a la estructura global del sistema, un nivel intermedio, por ejemplo, distribución de recursos y presupuestos y un nivel micro relacionado con la dinámica interna de las organizaciones.

El abordaje metodológico para la investigación en servicios de salud ha utilizado principalmente el enfoque cuantitativo; sin embargo, este enfoque ha dejado de lado una serie de aspectos interpretativos de la realidad, lo que ha producido, por ejemplo, que las reformas que buscan mejorar el sistema se encuentren con barreras y resistencias no previstas en su diseño.

Con el propósito de abordar los desafíos y problemas de salud con una perspectiva amplia y con la menor incertidumbre posible, ha surgido un enfoque metodológico en investigación que comparte la mirada cuantitativa y cualitativa a la vez, conocido en la actualidad como Método Mixto.

El objetivo de este ensayo es analizar los alcances y limitaciones de los métodos mixtos como diseño en la investigación en salud.

\section{MÉTODOS MIXTOS EN INVESTIGACIÓN}

La combinación de métodos cuantitativos y cualitativos en un solo es-

Claudio Muñoz

Poblete

Departamento de Medicina Interna, Universidad de La Frontera. cmunoz@ufro.cl tudio es cada vez más frecuente en la investigación en salud, a pesar de que sus aproximaciones al problema pertenecen a paradigmas diferentes (1). El interés demostrado por la combinación del uso de métodos cualitativos y cuantitativos puede ser atribuida a varias razones. Una de ellas es el aumento del rigor metodológico del enfoque cualitativo, que ha hecho 
que sea más aceptable para los investigadores formados dentro de un paradigma predominantemente cuantitativo; una segunda razón son las reconocidas contribuciones de la investigación cualitativa para el estudio y comprensión más profunda de la salud y la enfermedad (2). Esta situación es particularmente importante en los problemas de salud pública, donde las enfermedades de las poblaciones están vinculadas no solo a aspectos biomédicos, sino además a factores sociales, políticos y contextos económicos. La complejidad de los problemas de salud necesariamente requiere ampliar la mirada hacia lo epidemiológico y también lo social.

Si revisamos la historia de la investigación en servicios de salud observamos que ha estado centrada principalmente en el uso de metodologías cuantitativas. Pero hace ya más de una década que las investigaciones de tipo cualitativo se han incorporado como un aporte valioso en la comprensión de los problemas $(3,4)$.

Hay muchas maneras en las cuales diferentes métodos de investigación pueden ser combinados para aproximarnos al problema. Es importante hacer la distinción entre los estudios multimétodos y los estudios de métodos mixtos. En los primeros se utilizan diferentes métodos de colección y análisis de datos bajo un mismo paradigma de investigación, como por ejemplo, en un estudio cualitativo cuando se observa y a la vez se entrevistan individuos, o en el caso de estudios cuantitativos cuando se realiza una encuesta sobre las actitudes de los estudiantes y también se recolecta información de registros computacionales sobre ellos mismos. Por su parte, en los estudios mixtos existe un intento por reunir métodos provenientes de distintos paradigmas de investigación, por ejemplo conducir un estudio en que se apliquen entrevistas semiestructuradas en un pequeño grupo de estudiantes y también llevar a cabo una encuesta a gran escala.

Tashakkori y Teddie (5) van más allá y argumentan la necesidad de una clarificación, debido a que ellos ven la emergencia de los métodos mixtos como un "tercer paradigma", distinto a la perspectiva positivista de la investigación cuantitativa y distinta a la perspectiva contructivista de la investigación cualitativa. Sin embargo, a mi parecer esto requiere un mayor desarrollo conceptual para llegar a esta conclusión. Estos autores incluso proponen que más que hablar de "métodos mixtos" es más apropiado usar el término "modelo mixto", en parte debido a que el enfoque es aplicado más allá de los métodos utilizados en la investigación, pudiendo recaudar tanto o más información que la recopiladas por los enfoques de manera separada (6). Por lo tanto, es necesario clarificar en el método mixto lo que está siendo vinculado y de que manera se está haciendo, incluso el que pueda llegar a estar completamente integrado en un análisis único, como lo propone Caracelli y Greene (7).

Podemos resumir entonces que el enfoque mixto es un proceso que recolecta, analiza y vincula datos cuantitativos y cualitativos en un mismo estudio, para responder a un planteamiento del problema o para responder a preguntas de investigación. Como indica Tashakkori y Teddlie (5) un estudio mixto lo es en el planteamiento del problema, la recolección, análisis de los datos y el informe del estudio.

Julia Brannen (8) se pregunta, ¿cuáles son las razones para optar por el método mixto? Ella se refiere a las "tres P": paradigmáticas, pragmáticas y políticas. Primero, en las razones paradigmáticas, el método cualitativo y cuantitativo proviene de dos corrientes filosóficas distintas, una positivista, que supuestamente busca la verdad, y el otro más bien interpretativo, tratando también supuestamente de entender la realidad; sin embargo, todos reconocemos que tanto la verdad como la realidad están superpuestas. Por su parte si es interés de los investigadores abordar los problemas desde distintas dimensiones, podemos encontrarnos con un punto de vista macro relacionado con lo cuantitativo y generalizable, referido a patrones y tendencias de mayor escala que busca dar explicaciones estructurales, o desde la otra dimensión más bien micro e interpretativa, considerando la perspectiva subjetiva. No cabe duda que todos los investigadores sociales están interesados en entender los 
individuos insertos en la sociedad, por lo cual lograr trascendencia entre ambas dimensiones está implícito en las preguntas del investigador. Si cuestionamos incluso la objetividad del investigador cuantitativo podemos decir que este no es neutro pues tiene una perspectiva que determina desde qué quiere estudiar, cómo lo quiere hacer, e incluso es él quien interpreta los resultados, dando cuenta del componente subjetivo de este tipo de investigador

Segundo, existen varias razones pragmáticas que justifican el uso de métodos mixtos. Podemos dar algunos ejemplos: permiten acceder a poblaciones "ocultas" o no representadas en la sociedad tal como la entendemos (p.e. inmigrantes), o ser capaces de detectar temas que son sensibles para un método más que con otro; por ejemplo el análisis de conductas o comportamientos usando técnicas cualitativas, más apropiadas que los cuestionarios de autorreportes, utilizados en algunos casos más por razones económicas que metodológicas. Probablemente las razones pragmáticas de conducir investigaciones mixtas lo hace menos puristas con las ideas y métodos preconcebidos desde la mirada paradigmática, sin embargo, la razones pragmáticas que conducen ciertas investigaciones es que la vincula con fines prácticos o políticos, lo cual lo hace sumamente valioso para efectos de la toma de decisiones.

$\mathrm{Y}$ en tercer lugar, las razones políticas de conducir investigaciones mixtas es conectarla justamente con el ¿para quién? y ¿para qué? Debido a estas preguntas se puede decir que se encuentra más vinculada a las razones pragmáticas de la investigación que a las paradigmáticas. Ejemplo de esto son los estudios de justicia social, cuando queremos acceder a grupos “invisibles" para estudios cuantitativos a gran escala, o hacer explícitas las perspectivas de grupos oprimidos, sin acceso o representación.

Cuando la investigación mixta tiene lugar en el proceso investigativo social es importante discutir la manera en que se combina y analiza la información obtenida por ambos métodos. Existen cinco justificaciones para combinar la investigación cualitativa y cuantitativa:
1. Iniciación: el uso de un método ofrece las primeras luces para nuevas hipótesis, "tiene por objeto el descubrimiento de la paradoja y la contradicción, de nuevas perspectivas, de reformulación de preguntas o resultados de un método con las preguntas o los resultados de otro método" (9).

2. Complementariedad: "tiene por objeto la elaboración, la mejora, la ilustración y la aclaración de los resultados de un método apoyados con los resultados del otro" (9).

3. Desarrollo: "trata de utilizar los resultados de un método para ayudar a desarrollar o informar al otro método, donde el desarrollo es en el sentido general para métodos de muestreo y aplicación, así como la toma de decisiones" (9).

4. Expansión: "se pretende ampliar el alcance de la investigación” (9). Aquí el uso de un tipo de análisis de datos añade comprensión a lo adquirido por otra.

5. Triangulación: conocido también como convergencia, corroboración o correspondencia de los resultados a partir de distintos métodos. Se hace hincapié en la búsqueda de confirmación entre los datos cuantitativos y cualitativos (10).

Los estudios mixtos también podemos caracterizarlos en términos de los efectos de la combinación de métodos (11), para lo cual es importante entender la prioridad de los métodos dentro de un estudio, percibiendo si es el método cuantitativo el que predomina sobre el cualitativo o a la inversa, o bien la secuencia en que los métodos se utilizan (9).

\section{APLICACIÓN DE LA METODOLOGÍA MIXTA EN INVESTIGACIÓN DE SISTEMAS Y SERVICIOS DE SALUD (ISS)}

Al revisar la literatura relacionada con ISS las razones aludidas al uso de métodos mixtos se apegan bastante a lo descrito por los autores que han desarrollado el método; principalmente la vinculación es hecha entre la investigación cualitativa con ensayos controlados aleatorizados (ECA), las que 
han tenido un desarrollo importante en el Reino Unido, donde ha tenido bastante receptividad por parte de la comunidad (12). Los usos más destacados son, por ejemplo, aquellos en los cuales se permite evaluar las discrepancias aparentes de los resultados de investigaciones cualitativas y cuantitativas en la fase piloto de un estudio. También usados para facilitar la definición y direccionar preguntas de investigación; por ejemplo un estudio cualitativo puede ayudar a darnos una estructura conceptual al problema para poder abordar un estudio experimental de manera más íntegra, disminuyendo la incertidumbre al incorporar variables relevantes. El mejoramiento del diseño de ECA con el uso complementario de un estudio cualitativo puede proveer también un marco contextual al estudio, como por ejemplo, dar representatividad social a grupos con bajo empoderamiento.

En la agenda de las prioridades de la ISS han predominado los estudios económicos para apoyar la toma de decisión, dejando un abanico de problemas sin resolver. Cuando otros enfoques disciplinarios toman lugar para entender el funcionamiento de las organizaciones el panorama se hace más complejo; por lo mismo, si no consideramos la mutidimensionalidad de los problemas, la probabilidad de que las decisiones tomadas fracasen por insuficientes es alta.

Uno de los propósitos de usar los métodos mixtos en el ámbito de los sistemas y servicios de salud es buscar principalmente la integralidad para entender las complejidades del sistema sanitario. También el uso creciente de métodos mixtos ha permitido comprender el impacto de las prestaciones y organización de los servicios de salud, con un enfoque centrado en los procesos, así como en los resultados (13).

El enfoque mixto ofrece varias ventajas al contar con una variedad de observaciones derivadas de diferentes fuentes, tipos de datos y contextos, produce información más rica y variada, y aumenta la posibilidad de ampliar las dimensiones del proyecto de investigación, permitiendo una riqueza interpretativa y sentido de entendimiento.
Los métodos mixtos no son en sí mismos ni más ni menos válidos que cada enfoque específico de investigación y al igual que con cualquier investigación, la validez deriva más de la idoneidad, exhaustividad y eficacia con que esos métodos se aplican.

El nivel de desarrollo de los métodos mixtos en ISS ha sido lento pero progresivo y los propulsores para su desarrollo son de índole prácticos; en mi opinión las ventajas han quedado bastante claras en los párrafos anteriores. Veamos entonces a continuación algunas limitaciones de su uso.

Se han identificado varios factores que impiden a los investigadores que usan métodos mixtos ser capaces de reunir en un solo proyecto métodos cuantitativos y cualitativos; por ejemplo, los investigadores deben considerar de antemano que existen diferentes tiempos y ritmos en cada tipo de método. Esta variable puede ser afectada por el requerimiento del investigador o patrocinadores de la investigación, e incluso los editores de revistas, de apurar los procesos para la obtención de resultados, saltándose etapas relevantes del proceso de investigación mixta y en definitiva optar por presentar resultados de solamente uno ellos.

La propuesta metodológica y conceptual de juntar dos tipos de métodos en un proyecto presenta una dificultad importante en la etapa de análisis conjunto de datos cuantitativos y cualitativos, de manera de proporcionar un análisis fusionado. Una consideración que puede ayudar a la vinculación de los análisis es en primer lugar, no perder de vista la razón de la realización de investigaciones con métodos mixtos. El análisis de los contenidos de los proyectos indica que es muy común que los investigadores que usan métodos mixtos abandonen en la etapa final hacer este tipo de investigación por razones ajenas a sus fundamentos (9, 14). La dificultad de integración de los resultados de ambas métodos puede deberse a falta de experiencia o incapacidad del investigador. Una pregunta que platea Alan Bryman (15) y que trata de averiguar la real integración entre ambos métodos es: “ ¿ha sido mi comprensión de los hallazgos 
cuantitativos y cualitativos, sustancialmente mejorada por el hecho de tener resultados cualitativos y cuantitativos y he demostrado este enriquecimiento?" Si la respuesta es no, es difícil ver cómo el investigador puede haber efectuado un análisis integrado más allá de lo mínimo.

Otra limitación descrita por la literatura tiene que ver con deficiencias significativas en nuestra comprensión de la práctica de métodos mixtos, es decir, se ha prestado poca atención al hecho de que la investigación de métodos mixtos tiene que ser redactada. $\mathrm{La}$ presentación de los resultados, en vez de ser presentada como dos esferas separadas, debe mostrar un intercambio mutuo de información. El uso de la triangulación (10) a veces ha obstaculizado este proceso, enfrentando los datos y concentrándose en el grado en que las conclusiones se refuerzan mutuamente o entran en conflicto. El manejo conjunto de datos cualitativo y cuantitativo debe ser planteado como un complemento y no una competencia por identificar la verdadera versión de los acontecimientos.

Los sistemas y servicios de salud presentan complejos modelos organizacionales, financieros y operacionales instalados en los países de acuerdo a sus condiciones y capacidades, con el fin de ofrecer un bienestar sanitario óptimo a la población. Probablemente dada la complejidad para enfrentar los fenómenos y problemas en estas organizaciones es que las investigaciones cuantitativas han incorporado a los métodos cualitativos. Pareciera entonces que han sido los investigadores cuantitativos los que han reconocido que su aproximación a la realidad es aún insuficiente cuando lo hacemos únicamente desde una mirada positivista.

En conclusión, la investigación con métodos mixtos se compromete con la variedad de temas relevantes de la atención sanitaria. Puede ser debido a este motivo que se han ido instalando de manera progresiva en la comunidad internacional, interesada en comprender los fenómenos que involucra a los sistemas y servicios de salud. Finalmente, desde mi punto de vista trabajar con dos paradigmas de investigación solo se ha visto justificado por un sentido pragmático y político; aún queda pendiente como resolver los conflictos fundamentalistas y las barreras conceptuales que enfrenta la investigación cualitativa y la cuantitativa.

\section{REFERENCIAS}

1. Brannen J. Mixing Methods: qualitatuive and quantitative research. Aldershot, Ashgate. 1992.

2. Bryman A. Quantity and Quality in Social Research. London, Routledge. 1995.

3. Pope C, Mays N. Reaching the parts others methods cannot reach: an introduction to qualitative methods in health and health services research. British Medical Journal. 1995; 311: 42-45.

4. Johnstone PL. Mixed methods, mixed methodology health services research in practice. Qualitative Health Research. 2004, 14(2): 259-271.

5. Tashakkori A, Teddlie C. Handbook of Mixed Methods in Social and Behavioral Reseach. Thousand Oaks, CA: Sage. 2003.

6. Barbour RS. Mixing qualitative methods: Quality assurance or qualite quagmire? Qualitative Health Research. 1998; 8(3): 352-361.

7. Caracelli VJ, Greene JC. Crafting mixedmethod evaluation design. In: Greene JC, Caracelli VJ (Eds.) Advenles in mixedmethosd evaluation: The challenges and benefits of integrating diverse paradigms (pp. 61-72) San Francisco: Jossey-Bass. 1997.

8. Brannen J. Mixed methods research. Nacional Centre for Research Methods, Institute of Education, University London. 2005.

9. Greene JC, Caracelli VJ, Graham WF. Toward a conceptual framework for mixedmethod evaluation designs. Educational Evaluation and Policy Analysis. 1989; 11: 255-274.

10. Denzin N. Strategies of Multiple Triangulation. The Research Act: A theoretical Introduction to Sociological Methods. 1989.

11. Creswell JW, Clark VLP, Gutmann ML, Hanson WE. Advanced mixed methods research designs. In Handbook of mixed 
methods in social and behavioural research. Tashakkori A, Teddlie C. (Eds.) London: Sage Publications. 2003; 209-240.

12. O’Cathain A, Murphy E, Nicholl J. Why, and how, mixed methods research is undertaken in health services research in England: a mixed methods study. BMC Health Services Research. 2007; 7:85 doi:10.1186/14726963-7-85.

13. Fulop N, Allen P, Clarke A, Black N. Issues in studying the organisation and delivery of health services. In: Studying the organisation and delivery of health services Research Methods. London: Routledge. 2001.

14. Bryman A. Integrating quantitative and qualitative research: How is it done? Qualitative Research. 2006; 6: 97-113.

15. Bryman A. Barriers to Integrating Quantitative and Qualitative Research Alan Bryman University of Leicester, United Kingdom. Journal of Mixed Methods Research. 2007; 1(1): 8-22. 